\title{
A Multiphase Level Set Evolution Scheme for Aerial Image Segmentation Using Multi-scale Image Geometric Analysis
}

\author{
Wang Wei, Yang Xin, and Cao Guo \\ Institute of Image Processing and Mode Recognition, Shanghai Jiaotong University, \\ Shanghai, 200240, P.R. China \\ \{wangwei2002, yangxin, gcao_jn\}@sjtu.edu.cn
}

\begin{abstract}
This paper describes a new aerial images segmentation algorithm. The algorithm is based upon the knowledge of image multi-scale geometric analysis which can capture the image's intrinsic geometrical structure efficiently. The Contourlet transform is selected to represent the maximum information of the image and obtain the rotation invariant features of the image. A modified Mumford-Shah model is built to segment the aerial image by a necessary level set evolution. To avoid possible local minima in the level set evolution, we control the value of weight numbers of features in different evolution periods in this algorithm, instead of using the classical technique which evolve in a multi-scale fashion.
\end{abstract}

\section{Introduction}

Nowadays, with the development of sensor technology, the resolution of remotely sensed images has become higher, with more information being contained than before. Consequently, many remotely sensed image processing algorithms have appeared. Most of them are focused on the segmentation or classification of man-made objects.

The two main methods in the study of man-made object segmentation are: modelbased algorithms and feature-based algorithms.

Model-based algorithms include the works of $\mathrm{Jia} \mathrm{Li}^{[1]}$, A.L.Reno ${ }^{[2]}$, J.L.Solka ${ }^{[3]}$ etc. These algorithms can segment man-made objects precisely. However, it is very difficult to build a precise estimation model due to the complexity of remotely sensed images. Moreover, the computation of the estimated parameters of the model is inevitably complex and time-consuming.

Feature-based algorithms include the works of Mark.J Carlotto ${ }^{[4]}$, Stephen Levitt ${ }^{[5]}$ etc. These initial studies consider the low level features of the image. Recent studies integrate high level analysis of the features of color, texture, height and so on.

The remotely sensed images segmentation methodology proposed in this paper is based on the knowledge of image multi-scale geometric analysis, which can extract the features of the image efficiently. How to obtain the rotation invariant features is described in the paper. In order to classify the remotely sensed images, a modified Mumford-Shah model is introduced to integrate the rotation invariant features, while the level set method is responsible for the image evolution. 
This paper is organized as follows: Section 2 introduces the Contourlet transform and the feature extraction method which is based on the knowledge of image multiscale geometric analysis. Section 3 introduces the modified Mumford-Shah model. Section 4 elaborates on the new aerial images segmentation algorithm. The outputs of experiments are presented and illuminated in Section 5 and the conclusions of the paper are listed in Section 6.

\section{Feature Extraction Based on Image Multiscale Geometric Analysis}

\subsection{Image Multi-scale Geometric Analysis and Contourlet Transform}

The wavelet transform is widely used in many fields, but it still has some limitations. E.J.Candès [6] indicates that wavelets provide a very sparse representation for piecewise smooth 1-D signals but fail to do so for multi-dimensioned signals. Minh N. Do $^{[7]}$ compared 2-D separable wavelet transform with multi-scale geometric analysis. As we find in the Fig.1: Multi-scale geometric analysis is more efficient than wavelet transform because of those elongated shapes and multiple directions along the contour.

In 2003, Minh N.Do introduced the contourlet transform ${ }^{[8]}$ which can be regarded as a discrete version of the curvelet transform. It solved most of the problems that the curvelet transform had met with, but it still has a redundancy ratio of about $33 \%$. Although the crisp-contourlets ${ }^{[9]}$ were later generated to reduce the redundancy ratio, $\mathrm{DFB}^{[10]}$ applications still exists in the low-frequency component. Truong $\mathrm{T}$. Nguyen and Soontorn Oraintara ${ }^{[11]}$ developed the theory of multi-resolution DFB which can be uniformly and maximally decimated. They introduced the uniform DFB(uDFB) and the non-uniform DFB(nuDFB) in their paper .

In this paper, the contourlet transform is efficient enough to extract the features of the remotely sensed image since the features of aerial Images are mainly concentrated in the middle and high frequency component. The contourlet transform is briefly described as Fig.2.

The contourlet transform consists of the Laplacian pyramid and the DFB. The union can be described as pyramidal DFB(PDFB). In each scale decomposition, the

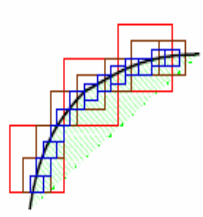

Wavelet

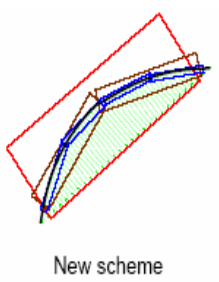

Fig. 1. Wavelet transform versus the new scheme ${ }^{[7]}$

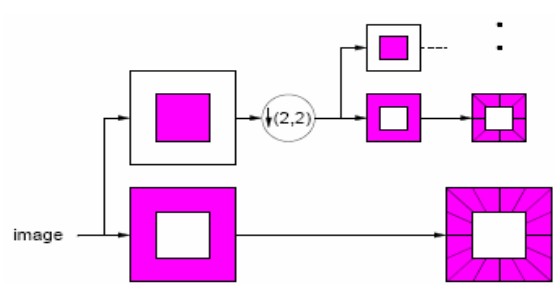

Fig. 2.The contoulet filter bank ${ }^{[7]}$ 
Laplacian pyramid separates the low-frequency component from the rest of the components, and then the DFB is applied to the rest.

\subsection{The Extraction of Rotation Invariant Features}

The features of aerial Images are mainly concentrated in the middle and high frequency components, while the low-frequency components usually contain the gray scale information. So we only need extract the features of the middle and high frequency components which we are interested in. The contourlet transform can meet our needs and avoid the complexities of the DFB brought upon by the UDFB and $\mathrm{nuDFB}^{[11]}$.

Manesh Kokare ${ }^{[12]}$ proposed a new rotationally invariant feature extraction method, in which the images are decomposed into different sub-bands by DT-CWT and DTRCWF, then the final rotation invariant wavelet features are obtained from those subbands. Referring to Manesh Kokare's method, the rotation invariant contourlet features can be extracted as follows:

To calculate the features of a certain point in a remotely sensed image, we select a block with a size of $16 \times 16$ or $32 \times 32$, with a certain point in the center of the block. Then, we decompose this block into three levels by the contourlet transform. As to the first two levels of contourlet decompositions, we use a three levels DFB decomposition to get an eight-directional frequency partitioning for each level; as to the final contourlet decomposition, the wavelet transform is used to obtain 4 different sub-bands. In the end, the targeted block is decomposed into 20 sub-bands, just as Fig 3 shows.

Then the rotation invariant contourlet features in each level can be calculated, supposing that $\mathrm{j}$ denotes $\mathrm{jth}$ level, the size of sub-band $w_{j}^{i}$ is $m \times n$, the feature of $w_{j}^{i}$ is calculated as follows:

$$
\begin{gathered}
E_{j}^{i}=\frac{1}{N M} \sum_{l=1}^{N} \sum_{k=1}^{M}\left|x_{j}^{i}(l, k)\right| \\
\mu_{j}^{i}=\frac{1}{N M} \sum_{l=1}^{N} \sum_{k=1}^{M} x_{j}^{i}(l, k) \\
\sigma_{j}^{i}=\left[\frac{1}{N \times M} \sum_{l=1}^{N} \sum_{K=1}^{M}\left(x_{j}^{i}(l, k)-\mu_{j}^{i}\right)^{2}\right]^{\frac{1}{2}}
\end{gathered}
$$

Where $E_{i}^{j}$ is the energy of $w_{j}^{i}, \sigma_{i}^{j}$ is the standard deviation of $w_{j}^{i}, \mu_{i}^{j}$ is the mean of $w_{j}^{i}, x_{i}^{j}(l, k)$ is the coefficients of $w_{j}^{i}$ located in $(l, k)$.

While the level $\mathrm{j}$ equal 1 or 2 , the rotation invariant features is given by (4), while the level $\mathrm{j}$ equal 3 , the rotation invariant features is given by (5). 


$$
\begin{gathered}
E_{j}=\frac{1}{8} \sum_{i=0}^{7} E_{j}^{i} \quad \sigma_{j}=\frac{1}{8} \sum_{i=0}^{7} \sigma_{j}^{i} \\
E_{3}=\frac{1}{2}\left(E_{3}^{1}+E_{3}^{2}\right) \quad \sigma_{3}=\frac{1}{2}\left(\sigma_{3}^{1}+\sigma_{3}^{2}\right)
\end{gathered}
$$

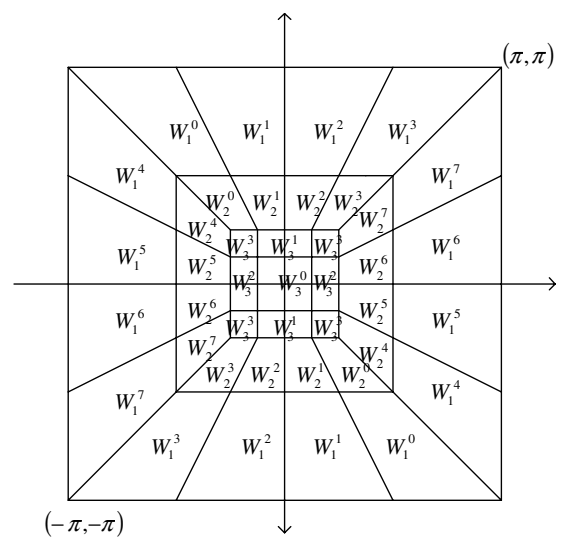

Fig. 3. Frequency partition of the three levels decompositions

The final six dimension rotation invariant features is given by

$$
\text { feature }=\left[\begin{array}{llllll}
E_{3} & E_{2} & E_{1} & \sigma_{3} & \sigma_{2} & \sigma_{1}
\end{array}\right] \bullet\left[\begin{array}{cccccc}
K_{1} & 0 & 0 & 0 & 0 & 0 \\
0 & K_{2} & 0 & 0 & 0 & 0 \\
0 & 0 & K_{3} & 0 & 0 & 0 \\
0 & 0 & 0 & K_{4} & 0 & 0 \\
0 & 0 & 0 & 0 & K_{5} & 0 \\
0 & 0 & 0 & 0 & 0 & K_{6}
\end{array}\right]
$$

Where $K_{1} \sim K_{6}$ are weight numbers.

\section{Mumford-Shah Model and the Modification}

The Mumford-Shah model ${ }^{[13]}$ is a commonly used model in image segmentation, based on this Chan and Vese proposed a multi-phase level set framework ${ }^{[14]}$ for image segmentation. In the piecewise constant case, $\mathrm{n}$ phases can be represented by $\mathrm{m}$ level set functions, where $\mathrm{m}=\log _{2} n$. In this framework, there exist interactions between each level set function, which will reduce the speed of evolution.

In order to speed up the aerial images segmentation algorithm, we still use $n$ instead of $\log _{2} n$ level set functions to represent $\mathrm{n}$ phases. This method avoids the interaction between different level set functions. However, it has brought about new 
problems, such as vacuum or overlapping points, which can be regarded as payment for pursuing fast segment algorithm. After finishing the evolution, we need to classify these points by a strategy that will be discussed in later chapters.

The active contour evolving method can combine other features besides the grey level features. Jean-Francois Aujol, Gilles Aubert, and Laure Blanc-Féraud ${ }^{[15]}$ presented a supervised classification model based upon a variational approach. The wavelet features are taken into consideration in this model. Cao Guo ${ }^{[16]}$ proposed a simplified Mumford-Shah model in which the features of fractal error metric and the DCT coefficients of texture edges are considered.

In the situation of supervised classification that the mean values of each region are pre-known, we can obtain the jth energy function as follows:

$$
\begin{aligned}
& F_{j}\left(C_{j}, c_{o}, c_{b j}\right)=u \cdot \text { Length }\left(C_{j}\right)+\lambda_{1} \cdot \int_{\text {inside }(C)} \mid \text { feature }-\left.\overline{\text { feature }}_{o j}\right|^{2} d x d y+ \\
& \lambda_{2} \cdot \int_{\text {outside }(C)}\left(\mid \text { feature }-\left.\overline{\text { feature }}_{b_{j}}\right|^{2}\right) d x d y
\end{aligned}
$$

Where feature are the six dimension features of point $(x, y), \overline{\text { feature }_{o j}}$ denotes the mean feature of the jth region, $\overline{\text { feature }}_{b j}$ is a changing value decided by the position $(x, y), \overline{\text { feature }}_{b_{j}}$ is selected from one of the $\mathrm{n}$ pre-known mean values except $\overline{\text { feature }_{o j}}$.

Function(7) can be represented in another form as follows:

$$
\begin{aligned}
& F_{j}\left(\phi_{j}, c_{o}, c_{b j}\right)=u \cdot \int_{\Omega} \delta\left(\phi_{j}\right)\left|\nabla \phi_{j}\right| d x d y+\lambda_{1} \cdot \int_{\Omega} \mid \text { feature }-\left.\overline{\text { feature }}{ }_{j}\right|^{2} H\left(\phi_{j}\right) d x d y+ \\
& \lambda_{2} \cdot \int_{\Omega}\left(\mid \text { feature }-\left.\overline{\text { feature }}_{b_{j}}\right|^{2}\right)\left[1-H\left(\phi_{j}\right)\right] d x d y
\end{aligned}
$$

Where $\phi_{j}$ is the jth level set function.

The associated Euler-Lagrange equations to (8) give the following expression:

$$
\left\{\begin{array}{l}
\overline{\text { feature }}_{o j}=\frac{\int_{\Omega} \text { feature }(x, y) H\left(\phi_{j}\right) d x d y}{\int_{\Omega} H\left(\phi_{j}\right) d x d y}, \\
\mid \text { feature }(x, y)-\left.\overline{\text { feature }_{b j}}\right|^{2}=\min \left(\mid \text { feature }(x, y)-\left.\overline{\text { feature }_{o i}}\right|^{2}\right), 1 \leq i \leq n, i \neq j ; \\
\frac{\partial \phi_{j}}{\partial t}=\delta\left(\phi_{j}\right)\left[u \nabla \cdot \frac{\nabla \phi_{j}}{\mid \nabla \phi_{j}}-\lambda_{1} \cdot\left[\text { feature }(x, y)-\overline{\text { feature }_{o j}}\right]^{2}+\lambda_{2} \cdot\left[\text { featurd }(x, y)-\overline{\text { feature }_{b j}}\right]^{2}\right] \\
\phi_{j}(0, x, y)=\phi_{0}(x, y) ;
\end{array}\right.
$$

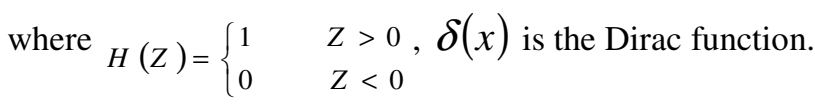


To avoid possible local minima in the level set evolution, one classical technique is to evolve in a multi-scale fashion ${ }^{[17]}$. The evolution result from the lower resolution is selected to be the initial contour of the next evolution in the higher resolution. Instead of using the classical technique referred to above, we control the value of $K_{1} \sim K_{6}$ in different evolution periods in this algorithm. In the beginning stages of the resolution, the features of lower resolution are applied with bigger weight. When the level set evolves into the more constant stages, the value of $K_{1} \sim K_{6}$ changes to ensure the features of higher resolution are applied with bigger weight. The changing weighting numbers will lead the geodesic flow to the correct position not only in the lower resolution but also in the higher resolution.

\section{Description of the Aerial Images Segment algorithm}

The aerial image segmentation algorithm proposed in this paper is a supervised method. The procedure of segmentation can be described as follows:

Step 1: First of all, select the representative sections of different classes from the aerial image and save these sections into the list.

Step 2: Set the weighting numbers $K_{1} \sim K_{6}$ to 1 , then calculate the norm feature $\overline{\text { feature }_{o i}}$ of each section in the list. Calculate the feature feature $(x, y)$ of every point in the aerial image. Save $\overline{\text { feature }_{o i}}$ as $\overline{\text { feature }_{o i}}$ sav and save feature as feature_sav.

Step 3: Referring to Chan and Vese ${ }^{[14]}$, initial closed curves in the aerial image are given in this algorithm, just as Fig 4(b) shows.

Step 4: The parameters are initially set as: $K_{1}=K_{4}=1.2, K_{2}=K_{5}=1.2$, $K_{3}=K_{6}=0.6$. Refresh the values of $\overline{\text { feature }_{o i}}$ and feature according to $\overline{\text { feature }}_{o \text { _ }}$ sav and feature_sav. The curve begins to evolve as described in the equation (9).

Step 5: When the difference between the two evolving steps is smaller than a predefined threshold as $T_{1}$, set the parameters as $K_{1}=K_{4}=0.6, K_{2}=K_{5}=1.2$, $K_{3}=K_{6}=1.2$. Refresh the values of $\overline{\text { feature }_{o i}}$ and feature again. Keep on the evolution.

Step 6: When the difference between two evolving steps is smaller than a predefined threshold as $T_{2}$, set the parameters as $K_{1}=K_{4}=0.6, K_{2}=K_{5}=1$, $K_{3}=K_{6}=1.4$. Refresh the values of $\overline{\text { feature }_{o i}}$ and feature again. Keep on the evolution.

Step 7: Update and evolve the level set function $\phi_{j}$ and check whether the criterion of termination is met or not. If the criterion of termination is met, the area inside the closed curves is the area of the jth class object. Start the $\phi_{j+1}$ evolution, repeat the steps of 4 7. 
Step 8: After all the level set functions evolution have finished, check the whole image to find the vacuum or overlapped points. Calculate the mean features of these points and their neighboring points. Classify these points to their nearest class in terms of the calculated mean feature $\overline{\text { feature }_{o i}}$.

Step 9: According to the result of evolution, differentiate each region using different colors.

\section{Experiment Results and Discussion}

In these experiments, the criterion of termination is met when the difference between two evolving steps is smaller than a pre-defined threshold as 0.015 or the evolution reach 20 times. Set the parameters as $T_{1}=0.45, T_{2}=0.15, \lambda_{1}=\lambda_{2}=0.2$.

The original aerial image with a size of $495 \times 385$ is shown in Fig 4(a), while the initial closed curves in the aerial image are shown in Fig 4(b).

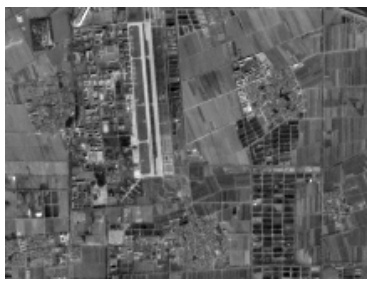

(a)

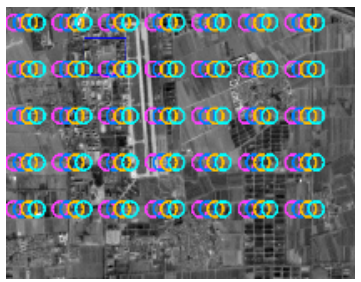

(b)

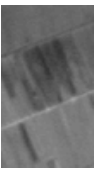

(c)

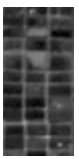

(d)

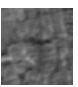

(e)

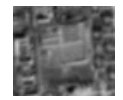

(f)

Fig. 4. The aerial image to be classified. (a) The aerial image. (b) Initial conditions. (c) (f) Different represent regions, the positions of these regions are $\{(253,71),(307,167)\}$, $\{(353,252),(397,344)\},\{(39,119),(83,167)\}$ and $\{(111,44),(170,96)\}$, respectively, on rectangular coordinates of the aerial image.

The supervised method is taken to segment this aerial image into four kinds of regions, for which the selected representative regions are shown in Fig 4(c) (f). The segmentation results are shown in Fig 5.

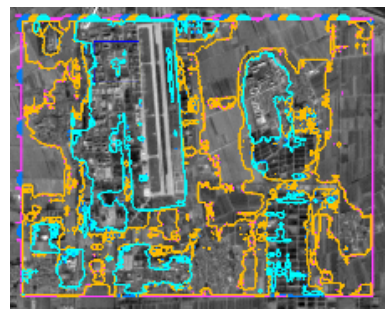

(a)

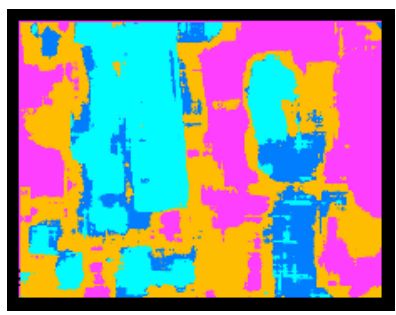

(b)

Fig. 5. Segmentation results. (a) Result of evolution. (b) Differentiate each region using different colors. 
The experiment results of segment aerial images with two and three classes are illustrated in Fig. 6 and Fig. 7, respectively. Satisfying experiment results are achieved by using the algorithm which is proposed in this paper.

More experiment results are shown as below:

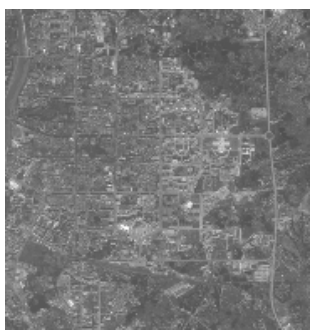

(a)

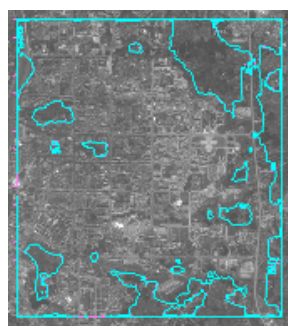

(b)

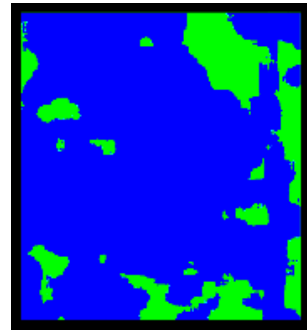

(c)

Fig. 6. Aerial image to be classified. The aerial image. (b) Result of evolution. (c) Differentiate each region by different colors.

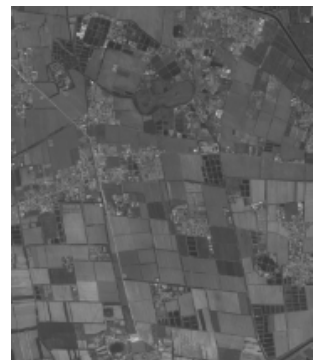

(a)

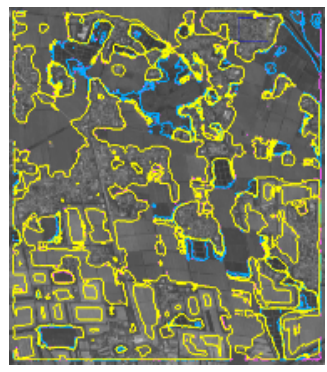

(b)

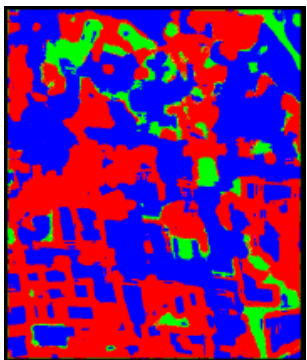

(c)

Fig. 7. Aerial image to be classified. (a) The aerial image. (b) Result of evolution. (c) Differentiate each region by different colors.

\section{Conclusion}

In this paper, a new supervised aerial images segmentation algorithm is presented. It is built on the basis of the multi-phase Mumford-Shah model. The rotation invariant contourlet features are obtained upon the knowledge of image multi-scale geometric analysis. In order to achieve a fast aerial images segmentation speed, several level set formulations are used to minimize the Mumford-Shah energy functions with contourlet features constraints. The proposed method is proven to be effective by the results of experiments.

\section{References}

1. Jia Li, Amir Najmi, Robert M.Gray. Image Classification by a Two-Dimensional Hidden Markov Model. IEEE Transactions on Signal Processing, 2000, 48(2), pp.517-533.

2. Reno, A.L., Booth, D.M. Using models to recognise man-made objects, Visual Surveillance.1999. Second IEEE Workshop on, 26 June 1999 pp.33 - 40. 
3. J.L.Solka, D.J.Marchette, B.C.Wallet. Identification of Man-Made Regions in Unmanned Aerial Vehicle Imagery and Videos. IEEE Transactions on PAMI, 1998, 20(8), pp.852-857.

4. Carlotto,M.J. Detecting Man-Made Features in SAR Imagery. Geoscience and Remote Sensing Symposium, 1996. IGARSS '96. 'Remote Sensing for a Sustainable Future. International, Volume: 1 , 27-31 May 1996.

5. Stephen Lebitt, Farzin Aghdasi. Texture Measures for Building Recognition in Aerial Photographs. Communications and Signal Processing, 1997. COMSIG '97., Proceedings of the 1997 South African Symposium on , 9-10 Sept, 1997.

6. E J Candès. Ridgelets :Theory and Applications. USA:Department of Statistics ,Stanford University ,1998.

7. Minh N. Do. Contourlets: A new directional multiresolution image representation. Conference Record of the Asilomar Conference on Signals, Systems and Computers, v 1, 2002, pp. 497-501.

8. Minh N. Do. Contourlets and Sparse Image Expansions. Proceedings of SPIE - The International Society for Optical Engineering, v 5207, n 2, 2003, pp. 560-570.

9. Y. Lu and M. N. Do, Crisp-contourlets: A critically sampled directional multiresolution image representation, in Proc. SPIE Conf. Wavelet Applications Signal Image Process. , San Diego, CA, Aug. 2003.

10. R. H. Bamberger and M. J. T. Smith, A filterbank for the directional decomposition of images: Theory and design, IEEE Trans. Signal Process., vol. 40, no. 7, pp. 882-893, Apr. 1992.

11. Truong T. Nguyen and Soontorn Oraintara. Multiresolution Direction Filterbanks: Theory, Design, and Applications. IEEE Transactions on Signal Processing, Vol.53, No. 10, October 2005, pp.3895-3905.

12. Manesh Kokare, P.K. Biswas and B.N. Chatterji. Rotation Invariant Texture Features Using Rotated Complex Wavelet For Content Based Image Retrieval. 2004 International Conference on Image Processing(ICIP), pp.393-396.

13. Mumford D, Shah J. Optimal approximation by piece wise smooth functions and associated variational problems. Communications on Pure and Applied Mathematics, 1989,42(5), pp.577-685.

14. Luminita A.Vese and Tony F.Chan, A Multiphase Level Set Framework for Image Segmentation Using the Mumford and Shah Model, International Journal of Computer Vision, 2002, 50(3), pp.271-293.

15. Jean-Francois Aujol, Gilles Aubert, and Laure Blanc-Féraud. Wavelet-Based Level Set Evolution for Classification of Textured Images. IEEE TRANSACTIONS ON IMAGE PROCESSING, Vol. 12, No. 12, DECEMBER 2003, pp. 1634-1641.

16. Cao Guo,Yang xin and Mao, Zhihong. A two-stage level set evolution scheme for manmade objects detection in aerial images Proceedings of the IEEE Computer Society Conference on Computer Vision and Pattern Recognition, v 1, Proceedings - 2005 IEEE Computer Society Conference on Computer Vision and Pattern Recognition, CVPR 2005, 2005, p 474-479.

17. Geiger, D., Gupta, A., Costa, L.A., and Vlontzos, J. 1995. Dynamic programming for detecting, tracking, and matching deformable contours. IEEE-PAMI, 17(3). 